\title{
Introduction to Volume 18, Issue 3/4
}

\section{Michael J. Cripps, University of New England}

The global pandemic continues to wreak havoc on all elements of society. Despite widespread vaccine access in the United States and many other countries, including access to boosters, COVID continues to infect in waves. Nearly two years out from the near-global lockdown of 2020, there is mounting evidence that pandemic-instruction in K-12 has not been good for many children's learning. Colleges and universities are seeing the effects of pandemic learning, as many first-year students seem unprepared for many of the basic expectations in college learning: attend class, complete homework, meet deadlines. Never mind the content. It is also clear that the stresses of the pandemic have negatively impacted many of our students' and colleagues' emotional health. I see it in my interactions, hear about it in meetings, and find it documented in both my institution's reports on student well-being and the popular press.

Many universities are requiring evidence of vaccination boosters for students, staff, and faculty. Mindful of the risks that Omicron poses to in-person instruction at residential campuses, an increasing number of colleges and universities are planning delayed starts to the Spring term, opening with a week or more of remote instruction, and/or ramping up testing, tracing, and isolation protocols. As recently as early November, I naively thought we might be turning a corner on the pandemic, which could enable us to focus more attention on meeting the emergent learning needs of young adults with COVID-induced high school educational experiences. Boosters were becoming widely available, and leadership at my university signaled a possible relaxation of some aspects of our mask mandate for the Spring term. Today, many of us are looking ahead to a likely third year of COVID protocols. As Steven Mintz (2021) put it at the end of the Fall term, "The pandemic will likely become endemic, with periodic, localized surges continuing to occur."

Beyond teaching and learning on campus, scholarship has also been affected by the continued pandemic. Where some professional organizations have been holding in-person academic conferences, many have not. The work involved in preparing and delivering classes in the pandemic, the work at home supporting K-12 children, and the complications involved in conducting research amid COVID protocols have negatively impacted many scholars' activity. Others have continued with their regular levels of research productivity, or so it seems. At some institutions, faculty have been able to pause the tenure clock or other high-stakes review. We are beginning to see submissions to Across the Disciplines that report WAC research conducted amid COVID, or with important implications for WAC work in the pandemic, including an article appearing in the current issue. I anticipate we will see more pandemic-inflected submissions in the coming year.

This double issue of Across the Disciplines features seven articles, as well as a book review. I am confident that individuals interested in research on WAC faculty development, writing in STEM, threshold concepts of writing, and writing transfer will find much of value. Two articles take up the central WAC matter of faculty development: Elisabeth Miller et al. (2022) offer important insights into ways that faculty members' direct experiences with writing shape their approaches to teaching with writing; Amy Mecklenburg-Faenger et al. (2022) share a model of an online, asynchronous WAC faculty seminar developed at their institution and report data on disciplinary faculty members' experiences with the model. Three contributions engage

\section{Across the Disciplines}

wac.colostate.edu/atd

A Journal of Language, Learning and Academic Writing

ISSN 554-8244

https://doi.org/10.37514/ATD-J.2022.18.3-4.01

Across the Disciplines is an open-access, peer-reviewed scholarly journal published on the WAC Clearinghouse and supported by Colorado State University and Georgia Southern University. Articles are published under a Creative Commons BY-NC-ND license (Attribution-NonCommercial-NoDerivs) ISSN 1554-8244. Copyright (c) 1997-2022 The WAC Clearinghouse and/or the site's authors, developers, and contributors. Some material is used with permission. 
the important, challenging issue of writing transfer, including one that foregrounds undergraduates' acquisition of threshold concepts for writing: Wendy Olson and Dave Kim examine far transfer by analyzing the junior writing portfolios of engineering students; John Whicker explores upper-level students' perceptions of the value of the knowledge they learned in FYC; and Enrique Paz examines the ways a vertical writing curriculum in geology develops and reinforces key threshold concepts of writing. Four of the contributions to this issue offer important insights into WAC/WID and STEM education. In addition to Paz's contribution on threshold concepts, Olson and Kim's article on transfer, Miller et al.'s study of faculty development, Tereza Kramer et al. (2022) explore a vertically integrated approach to peer review in a kinesiology program. Rebecca Hallman Martini, in a study of disciplinary faculty members' use of Writing in the Professions (WIP) assignments, invites the field to consider the value of WIP curricula that share affinities with both WAC/WID and business and technical communication. Intriguingly, and from very different angles, Paz, Hallman Martini, and Kramer et al. offer important insights into relationships between writing development and students' emerging professional identities. Rounding out the issue, Andrea Williams (2022) provides an insightful review of William Germano and Kit Nicholls' (2020) Syllabus: The Remarkable, Unremarkable Document that Changes Everything.

In our first article, Elisabeth Miller, Kathleen Daley Weisse, and Bradley Hughes (2022) explore WAC faculty seminar participants' beliefs about writing and the ways those beliefs inform disciplinary instructors' approaches to teaching with writing. On the surface, the project's empirical focus on the WAC seminar may seem to offer a "back to basics" approach to WAC research, but the contributions are hardly basic. Miller et al. shed important light on the ways that seminar participants develop assignments and point to the value of using a constructivist framework that treats instructors as learners. As the title forecasts, "Getting Personal" argues that personal experiences with writing inform disciplinary instructors' values and beliefs, which contribute significantly to the assignments they develop, the justifications they offer in peer-review workshops, and their interactions with other workshop participants. While Miller et al.'s findings will likely be of interest to anyone involved in WAC faculty development, given the disciplinary backgrounds and experience levels of the seminar participants, their findings will be of particular interest to those working with faculty in STEM and with teaching assistants.

As WAC faculty development is central to strengthening disciplinary faculty members' practices in teaching with writing, it is fitting that our second article offers additional insights into the WAC faculty seminar. Amy Mecklenburg-Faenger, Brandi Handley, and Emily Donnelli-Sallee (2022) invite readers to consider models for faculty development at institutions less commonly represented in the WAC scholarship, including those offering primarily online writing-intensive courses. "Making WAC Accessible" shares the Park University WAC program directors' efforts to develop and facilitate a meaningful four-week, asynchronous WAC workshop delivered to geographically dispersed full- and part-time faculty who teach online and within an enterprise model that affords limited curricular and pedagogical flexibility for instructors. Even as it operates in less-than-ideal circumstances for a WAC program, Park's asynchronous approach is not structured on self-paced tutorials. Rather, it treats the seminar as a recognizably complete four-week, asynchronous WAC workshop, with specific reading, discussion, and writing tasks for participants. While much of their model aligns with what one often finds in traditional face-to-face WAC seminars, the authors also explore important differences. The curriculum and data from faculty participants in "Making WAC Accessible" will interest WAC program administrators. The workshop the authors developed and share predates the emergence of COVID, but their contribution may be particularly timely as the pandemic continues to disrupt higher education.

Wendy Olson and Dave Kim (2022) focus attention on elements of an institution's vertical writing curriculum and engineering students' writing development in "An Exploratory Study of Far Transfer." Their contribution reports results from a genre analysis of undergraduate engineering students' junior writing portfolios (JWPs), supplemented with data from a focus group with engineering students. The authors draw from Yancey et al.'s (2014) framework for categorizing efforts at transfer and find support for 
the value of that framework for recognizing writers' ability to integrate prior learning into new writing situations. Although Olson and Kim identify their study as "exploratory," their findings point to audience awareness and style as two areas where transfer may be particularly challenging. As the authors note, effective communication is an important accreditation criterion for engineering programs, which may establish an exigence for engineering programs to focus attention on their students' writing development. Coming at the question of transfer from a WID perspective, Olson and Kim draw attention to the opportunities their findings create for faculty development, particularly among faculty and TAs teaching writing-in-the-major courses.

Also focused on writing transfer, John $\mathrm{H}$. Whicker's contribution reports results from a preliminary study that examines what students in upper-level writing-intensive courses remember learning in FYC and whether they found such knowledge helpful beyond the course. In addition to providing a valuable review of the literature on Writing About Writing (WAW) and Teaching for Transfer (TFT) approaches to FYC, Whicker adds to our knowledge of the potential long-term value of at least some elements of these approaches. Importantly, he also calls for transfer research that pushes beyond a "binary division of general and specific knowledge" (p. 288) and considers the possibility that transfer may operate at a "meso-level' of generality" (p. 300). The institutional context for the study, particularly the recent development and implementation a two-semester FYC sequence informed by WAW and TFT, enabled Whicker to compare responses from students who had previously completed the WAW-TFT FYC with those who had not. Although most of the students in the study reported finding at least some knowledge learned in FYC to be useful in upper-level courses-and many identified conceptual knowledge of genre and discourse communities as helpful-Whicker finds that students who had completed the redesigned FYC course with WAW and TFT elements expressed a more sophisticated sense of the concepts.

Our fifth article, "How Timing and Authority in Peer Review Impact STEM Students," reports findings from a comparative study of contributions that a "Writing Circles" (Circles) approach to peer review makes to kinesiology students' writing and critical thinking. Tereza Kramer et al. (2022), coming from a WID perspective, examine the impact of peer review on writing, as well as students' experience of peer review. They study the impact of Circles in both lower- and upper-level courses by scoring samples from pre- and post-implementation iterations of two key courses in a kinesiology curriculum. The authors find that in lower-level courses, the use of Circles yielded higher scores on outcomes associated with writing in the writing process. In upper-level courses, the inclusion of Circles had the largest impact on critical thinking learning outcomes. Interestingly, while scores on writing and on writing process seem to not to improve over those in the lower-level course, scores on critical thinking are considerably higher in the upper-level implementation of Circles. Additionally, they find that upper-level students see their peers more as disciplinary authorities qualified to offer feedback, which may help to explain gains in critical thinking. Individuals eager to get more from their peer review practice will benefit from reading Kramer et al.

Enrique Paz's (2022) contribution offers important insights into threshold concepts of writing and WID through his study of the impact of a geology program's vertical writing curriculum on student development. Drawing from situated learning theories and key threshold concepts of writing, "Changing Conceptions of Writing through Situated Activity in a Geology Major" brings student focus group and individual interview data together with an analysis of the ways that writing and writing instruction are embedded in the geology curriculum. Paz finds that students in the program come to internalize two threshold concepts in particular: writing mediates disciplinary activity, and writing constructs professional identity. He claims that two features of the geology curriculum play key roles in this development. First, the program situates writing within the work that geological communities perform. Second, by involving students in professional geological communities, the program encourages majors to recognize writing as a component of their disciplinary identity. As Paz notes, "Writing in this curriculum is not a separate object intersecting with geological activity but rather an operationalized tool in services of the community's motives" (p. 342). His article will be of particular interest to WAC program administrators working with programs on ways to 
integrate writing into their curricula, scholars looking for empirical studies of either threshold concepts or situated learning, and individuals looking to move writing more to the center of a STEM curriculum.

In our final article, Rebecca Hallman Martini advocates for greater attention to Writing in the Professions (WIP) approaches within WAC/WID. Exploring ways that WIP curricula can operate at the intersection of business and technical communication and academic writing genre approaches to WAC/WID, Hallman Martini argues that authentic WIP curricula engage students in professional genres and involve them in writing for authentic audiences that go beyond the instructor or the university. She reports results from open-ended interviews with ten faculty from a range of disciplines and finds that four key concepts for WIP models emerge from the data: composing in multiple modes; working collaboratively in teams; clarity in writing; and communicating to non-expert audiences. Also valuable in Hallman Martini's article is her discussion of the role of the writing program and, especially, WAC fellows in encouraging and supporting some of the WIP practices that faculty find valuable. Hallman Martini's contribution can be productively read alongside Paz's article, particularly by individuals eager to cultivate in students a sense that writing is integral to their disciplinary or professional identities.

Even as the pandemic poses significant challenges, we are excited by what ATD has in store for 2022, and beyond. Concurrent with the publication of this issue, we are sharing a call for proposals for a special issue on ePortfolios and Writing in the Disciplines. Guest edited by Christopher Basgier, Helen Chen, and Amy Cicchino, we anticipate this issue, currently scheduled for publication in late 2023, will bring the WAC/WID and ePortfolio communities closer together and stimulate conversations about high-impact practices, equity, assessment, and multimodal writing. This coming summer, we will publish a special issue on STEM and WAC guest edited by Erin Beaver, Brian Hendrickson, and Justin Nicholes. We also have many promising and important articles in various stages of the review process, including some that examine WAC work amid COVID. I encourage scholars whose work is relevant to the mission of ATD to consider sending manuscripts our way. And, as always, we are open to conversations with individuals seeking to propose a themed special issue of the journal.

\section{References}

Hallman Martini, Rebecca. (2022, February 18). More useful beyond college?: The case for a writing in the professions curriculum in WAC/WID. Across the Disciplines, 18(3/4), 346-363. https://doi.org/10.37514/ATD-J.2022.18.3-4.08

Kramer, Tereza Joy, Zeccardi, Joe, Emhoff, Chi-An W., Williams, Claire, Dunn, Robin J., \& Rose, Joshua. (2022, February 18). How timing and authority in peer review impact STEM students: A comparative assessment of writing and critical thinking in kinesiology courses. Across the Disciplines, 18(3/4), 305-319. https://doi.org/10.37514/ATD-J.2022.18.3-4.06

Mecklenburg-Faenger, Amy, Handley, Brandi, \& Donnelli-Sallee, Emily. (2022, February 18). Making WAC accessible: Reimagining the WAC faculty workshop as an online asynchronous course. Across the Disciplines, 18(3/4), 244-264. https://doi.org/10.37514/ATD-J.2022.18.3-4.03

Miller, Elisabeth L., Weisse, Kathleen Daly, \& Hughes, Bradley. (2022, February 18). Getting personal: The influence of direct personal experience on disciplinary instructors designing WAC assignments. Across the Disciplines, 18(3/4), 221-243. https://doi.org/10.37514/ATD-J.2022.18.3-4.02

Mintz, Steven. (2021, December 20). "10 takeaways from the Fall 2021 semester." Higher Ed Gamma. Inside Higher Ed. https://www.insidehighered.com/blogs/higher-ed-gamma/10-takeaways-fall-2021-semester

Olson, Wendy, \& Kim, David. (2022, February 18). An exploratory study of far transfer: Understanding writing transfer from first-year composition to engineering writing-in-the-major courses. Across the Disciplines, 18(3/4), 265-283. https://doi.org/10.37514/ATD-J.2022.18.3-4.04

Paz, Enrique. (2022, February 18). Changing conceptions of writing through situated activity in a geology major. Across the Disciplines, 18(3/4), 320-345. https://doi.org/10.37514/ATD-J.2022.18.3-4.07 
Whicker, John H. (2022, February 18). “Types of writing," levels of generality, and “what transfers?” upper-level students and the transfer of first-year writing knowledge. Across the Disciplines, 18(3/4), 284-304. https://doi.org/10.37514/ATD-J.2022.18.3-4.05

Yancey, Kathleen Blake, Robertson, Liane, \& Taczak, Kara. (2014). Writing across contexts: Transfer, composition, and sites of writing. Utah State University Press.

\section{Contact Information}

Michael J. Cripps, Ph.D.

Professor of Rhetoric \& Composition

Director of Composition

University of New England

Email: mcripps@une.edu

\section{Complete APA Citation}

Cripps, Michael J. (2022, February 18). Introduction to Volume 18, Issue 3/4. Across the Disciplines, 18(3/4), 216-220._216-220. https://doi.org/10.37514/ATD-J.2022.18.3-4.01 